Bundesgesundheitsbl 2019 - 62:238-246 https://doi.org/10.1007/s00103-019-02917-x Online publiziert: 19. Februar 2019

(c) Der/die Autor(en) 2019

CrossMark

Thomas Lampert · Jens Hoebel

Fachgebiet Soziale Determinanten der Gesundheit, Robert Koch-Institut, Berlin, Deutschland

\title{
Sozioökonomische Unterschiede in der Gesundheit und Pflegebedürftigkeit älterer Menschen
}

Erkrankungen und Beschwerden sowie funktionellen Einschränkungen und Behinderungen betroffen sind als Personen mit höherem sozioökonomischen Status. Zudem treten diese in der niedrigen Statusgruppe oftmals früher im Leben auf und sind mit weitreichenderen Auswirkungen auf das Wohlbefinden, die Lebensqualität und die soziale Teilhabe verbunden.

Den deutlichsten Ausdruck erfährt diese gesundheitliche Ungleichheit im mittleren Lebensalter. Die vorliegenden Forschungsbefunde sprechen aber dafür, dass auch im höheren Lebensalter sozioökonomische Unterschiede in der Gesundheit und der ferneren Lebenserwartung bestehen [6-9]. Dies scheint zumindest für die Altersspanne zwischen dem 65. und 80. Lebensjahr zu gelten. Studien, die 80 - oder 85 -jährige und ältere Menschen betrachten, kommen zum Teil zu dem Ergebnis, dass die sozioökonomischen Unterschiede schwächer ausfallen bzw. nicht mehr festzustellen sind [10-12]. Zurückgeführt wird dies unter anderem darauf, dass Personen mit niedrigem sozioökonomischen Status einem höheren vorzeitigen Sterberisiko unterliegen, also zu einem größeren Anteil gar nicht alt werden, und die vorzeitige Sterblichkeit vor allem Personen betrifft, die sozial und gesundheitlich am stärksten benachteiligt bzw. belastet sind („selective survival“; [13]). Außerdem wird davon ausgegangen, dass mit zunehmendem Alter biologische Alternsprozesse für das Krankheitsrisiko an Bedeutung ge- winnen und soziale Einflüsse zumindest zum Teil überlagern („age-as-a-leveler“; $[12,14,15])$.

Im Folgenden wird auf Basis für Deutschland vorliegender Daten die gesundheitliche Ungleichheit in der 65-jährigen und älteren Bevölkerung beschrieben. Das Interesse gilt dabei zunächst chronischen Erkrankungen, funktionellen Einschränkungen in der Alltagsaktivität sowie der subjektiven Gesundheit. Anschließend werden sozioökonomische Unterschiede in Bezug auf die Pflegebedürftigkeit und pflegerische Versorgung betrachtet. Zu Beginn wird kurz auf die Entwicklung und Verteilung des Armutsrisikos in der älteren Bevölkerung Deutschlands eingegangen.

\section{Armut und soziale Ungleichheit in der älteren Bevölkerung}

In Deutschland leben gegenwärtig 82,8 Mio. Menschen, von denen rund 17,7 Mio. 65 Jahre oder älter sind [16]. Den aktuellen Bevölkerungsvorausberechnungen zufolge wird der Anteil der 65-Jährigen und Älteren von derzeit $16 \%$ auf $22 \%$ im Jahr 2020 steigen. Ein Grund hierfür ist der kontinuierliche Anstieg der Lebenserwartung, der sich in allen Altersgruppen beobachten lässt, in den älteren Bevölkerungsgruppen aber überproportional ausfällt. Infolgedessen nimmt auch die Zahl der Hochbetagten zu. Geht man von der gegenwärtigen Bevölkerungsentwicklung aus, dann könnte sich die Zahl der 80-Jährigen und Äl- 


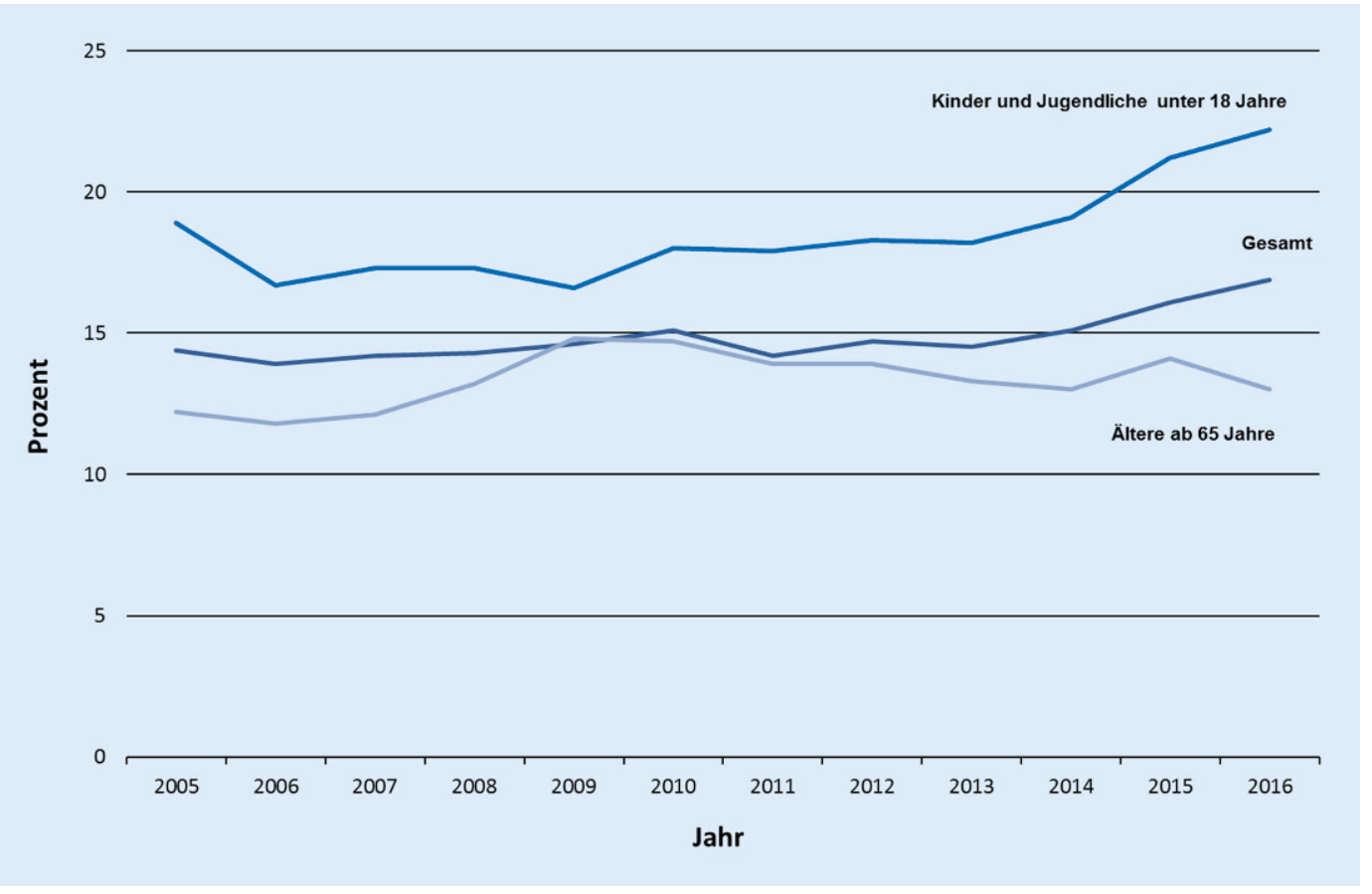

Abb. $1 \triangleleft$ Zeitliche Entwicklung des Armutsrisikos in Deutschland. Datenquelle: SOEP 2005-2016 teren in den nächsten zwei Jahrzehnten von 5,2 Mio. auf 7,8 Mio. erhöhen [17].

Nach dem Sozio-oekonomischen $\mathrm{Pa}$ nel (SOEP), einer großen Haushaltsbefragung, die jährlich vom Deutschen Institut für Wirtschaftsforschung durchgeführt wird, lebten im Jahr 2016 etwa $13 \%$ der 65 -Jährigen und Älteren in Armut oder waren von Armut bedroht (- Abb. 1). Die sogenannte Armutsrisikoquote bezieht sich auf den Anteil der Bevölkerung, der über ein Nettoäquivalenzeinkommen von weniger als $60 \%$ des gesellschaftlichen Mittelwertes verfügt; für einen Einpersonenhaushalt sind das zurzeit 1054€ [18]. Die Armutsrisikoquote der Älteren liegt damit unter dem Durchschnitt und deutlich niedriger als bei Kindern und Jugendlichen sowie jungen Erwachsenen, die überproportional häufig einem Armutsrisiko ausgesetzt sind. Die Daten des SOEP machen aber auch deutlich, dass das Armutsrisiko älterer Menschen in den letzten Jahren zugenommen hat, was unter anderem darauf zurückzuführen ist, dass aktuell Geburtsjahrgänge das Rentenalter erreichen, in denen mehr Menschen brüchige Erwerbsbiografien aufweisen und zumindest in bestimmten Gruppen, z.B. den Selbstständigen und den Geringqualifizierten, eine schlechtere Altersvorsorge besteht $[19,20]$.
Dies verdeutlichen auch die Zahlen des Mikrozensus des Statistischen Bundesamtes aus dem Jahr $2013 \mathrm{zu}$ bevölkerungsgruppenspezifischen Unterschieden in der Armutsbetroffenheit der älteren Bevölkerung (• Abb. 2). Während in der 65-jährigen und älteren Bevölkerung etwa $25 \%$ der Personen mit geringer beruflicher Qualifikation einem Armutsrisiko unterliegen, sind es von den Personen mit mittlerer und hoher beruflicher Qualifikation lediglich 12\% bzw. 6\%. Die Daten zeigen außerdem, dass Frauen, Personen mit Migrationshintergrund sowie Personen, die alleine leben, ein erhöhtes Armutsrisiko haben [21].

Daneben ist zu berücksichtigen, dass ältere Menschen über höhere Vermögen verfügen. Beispielsweise liegt der Anteil der Personen, die aus ihrem Vermögen jährliche Einkünfte über $5000 €$ beziehen, in der 65-jährigen und älteren Bevölkerung bei $11 \%$ im Vergleich zu 9\% und $6 \%$ in der 50 - bis 64-jährigen bzw. der 25- bis 49-jährigen Bevölkerung [18]. Auf der anderen Seite müssen viele ältere Menschen einen erheblichen Anteil ihres Vermögens für die Pflege und Unterbringung in einer Alteneinrichtung aufbringen. Zusammengenommen verdeutlichen diese Zahlen, dass das Einkommen und das Vermögen in der älteren
Bevölkerung sehr ungleich verteilt sind, was sich in den Lebensbedingungen und sozialen Teilhabechancen widerspiegelt und auch im Hinblick auf die Gesundheit von großer Bedeutung ist.

\section{Gesundheitliche Ungleichheit im höheren Lebensalter}

Aussagen zu sozioökonomischen Unterschieden in Bezug auf die Gesundheit und das Krankheitsrisiko im höheren Lebensalter sind unter anderem auf Basis von Daten der Studie zur Gesundheit in Deutschland aktuell (GEDA), die das Robert Koch-Institut regelmäßig im Rahmen seines Gesundheitsmonitorings durchführt, möglich. Nach den Daten aus den Jahren 2014/2015 berichten 62,8\% der 65-jährigen und älteren Männer und $64,8 \%$ der gleichaltrigen Frauen mit niedrigem sozioökonomischen Status einen mittelmäßigen, schlechten oder sehr schlechten allgemeinen Gesundheitszustand, während dies lediglich auf 36,3 \% der Männer und 35,7\% der Frauen aus der hohen Statusgruppe zutrifft (- Abb. 3 und 4).

Vergleichbare Unterschiede zwischen den Statusgruppen sind hinsichtlich gesundheitlich bedingter Aktivitätseinschränkungen im Alltag zu beobachten. Die hier ausgewiesenen Anteile 
beziehen sich auf Personen, die aufgrund ihrer Gesundheit „stark“ oder „mäßig“ in der Bewältigung alltäglicher Aufgaben eingeschränkt sind. Wie beim allgemeinen Gesundheitszustand so gilt auch für Alltagseinschränkungen, dass die Unterschiede zwischen Männern und Frauen der niedrigen und hohen Statusgruppe stark ausgeprägt sind. Darüber hinaus zeigen sich hinsichtlich beider Gesundheitsindikatoren Unterschiede zwischen der niedrigen und mittleren sowie der mittleren und hohen Statusgruppe, sodass davon gesprochen werden kann, dass Beeinträchtigungen des allgemeinen Gesundheitszustandes bzw. der Alltagsaktivität umso häufiger sind, je niedriger der sozioökonomische Status ist.

Geringer ausgeprägt sind die sozioökonomischen Unterschiede im Vorkommen mindestens einer chronischen Erkrankung. Zu berücksichtigen ist dabei, dass sich die zugrunde liegende Frage auf jegliche chronische Erkrankungen und Beschwerden bezieht, unabhängig von Schweregrad und Krankheitsfolgen. Deutlichere Unterschiede zwischen den Statusgruppen sind festzustellen, wenn spezifische, mit Einschränkungen für die Alltagsaktivität und Lebensqualität einhergehende Erkrankungen betrachtet werden. So beträgt z. B. die Lebenszeitprävalenz der koronaren Herzkrankheit bei 65-jährigen und älteren Männern mit niedrigem sozioökonomischen Status 30,8\%, während der Vergleichswert für Männer mit hohem sozioökonomischen Status bei 22,4\% liegt. Bei Frauen dieser Altersgruppe sind die Unterschiede zwischen den Statusgruppen mit $22,9 \%$ gegenüber $11,1 \%$ noch größer. Dass sie Diabetes mellitus haben, trifft auf 22,2\% der Männer aus der niedrigen und 17,2\% der Männer aus der hohen Statusgruppe zu. Bei Frauen ist die Differenz wiederum größer und beläuft sich auf $26,7 \% \mathrm{zu} 9,0 \%$ [22].

In den GEDA-Studien 2010 und 2012 wurden zudem verschiedene Fragen zum Befinden und zur Stimmungslage gestellt, um das seelische Wohlbefinden der Befragten aus ihrer Perspektive zu erfassen und damit einen zentralen Bereich der gesundheitsbezogenen Lebensqualität abzubilden. Es wurde unter anderem

Bundesgesundheitsbl 2019.62:238-246 https://doi.org/10.1007/s00103-019-02917-x

(c) Der/die Autor(en) 2019

\section{T. Lampert · J. Hoebel}

\section{Sozioökonomische Unterschiede in der Gesundheit und Pflegebedürftigkeit älterer Menschen}

\section{Zusammenfassung}

Angesichts der weiterhin steigenden

Lebenserwartung stellt sich die Frage, in welchem Gesundheitszustand die hinzugewonnenen Lebensjahre verbracht werden können. Die Forschungsbefunde zu sozialen Unterschieden in der Gesundheit und Lebenserwartung legen nahe, dass diese Frage für verschiedene Bevölkerungsgruppen unterschiedlich zu beantworten ist. Denn auch nach dem 65. Lebensjahr gilt, dass Personen mit niedrigem sozioökonomischen Status häufiger von chronischen Erkrankungen und Beschwerden, funktionellen Einschränkungen der Alltagsaktivität sowie Beeinträchtigungen des Wohlbefindens und der gesundheitsbezogenen Lebensqualität betroffen sind. Da aktuell noch ein erheblicher Anteil der Personen mit niedrigem sozioökonomischen Status vorzeitig stirbt, könnte die Ausweitung der Lebenszeit, die für alle Bevölkerungsgruppen zu beobachten ist, dazu führen, dass sich künftig die sozialen Unterschiede in der Gesundheit im höheren Lebensalter noch stärker abzeichnen. Damit ist die Herausforderung verbunden, bei Maßnahmen und Programmen, die darauf zielen, die Gesundheit älterer Menschen zu verbessern und Pflegebedürftigkeit vorzubeugen, die Belange sozial benachteiligter Bevölkerungsgruppen in besonderem Maße zu berücksichtigen.

Schlüsselwörter

Armut · Soziale Ungleichheit · Gesundheitliche Ungleichheit · Gesundheit im Alter . Pflegebedürftigkeit

\section{Socioeconomic differences in health and need for care among the elderly}

\section{Abstract}

In view of the continuing increase in life expectancy, the question arises as to the state of health in which these years of gained life can be spent. The research findings on social differences in health and life expectancy suggest that this question has to be answered differently for different population groups. Even after the age of 65 , persons with a low socioeconomic status are more likely to be affected by chronic illnesses and complaints, functional limitations of everyday activities, as well as impairments of well-being and health-related quality of life. With a significant proportion of people with low socioeconomic status still dying prematurely, the increase in lifetime that is observed for all population groups could increase the social gap in health in later life. This implies the challenge of giving special attention to the needs of socially disadvantaged groups in policies and programs aiming to improve the health of older people and preventing the need for care.

\section{Keywords}

Poverty - Social inequality · Health inequality . Health in older age $\cdot$ Long-term care danach gefragt, wie oft sich die Befragten in den letzten vier Wochen vor der Befragung „voller Leben“, „ruhig und gelassen“ sowie „glücklich“ fühlten. Die Angaben unterscheiden sich deutlich zwischen Personen mit niedrigem, mittlerem und hohem Sozialstatus. Unter Männern und Frauen im Alter ab 65 Jahren liegen die Anteile derer, die sich in den vergangenen vier Wochen „meistens“ oder „immer“ voller Leben, ruhig und gelassen bzw. glücklich fühlten, jeweils in der hohen Sozialstatusgruppe am höchsten und in der unteren Sozialstatusgruppe am niedrigsten (•Tab. 1). Auch bei statistischer Kontrolle für Altersunterschiede bleiben diese statusspezifischen Unterschiede im Wohlbefinden älterer Menschen zuungunsten derer mit niedrigem sozioökonomischen Status bestehen [22].

Damit übereinstimmende Ergebnisse liegen aus dem SOEP vor. Dies kann z. B. anhand des Indikators „Subjektive Gesundheit - weniger gut oder schlecht" verdeutlicht werden, der für die Armuts- und Reichtumsberichter- 


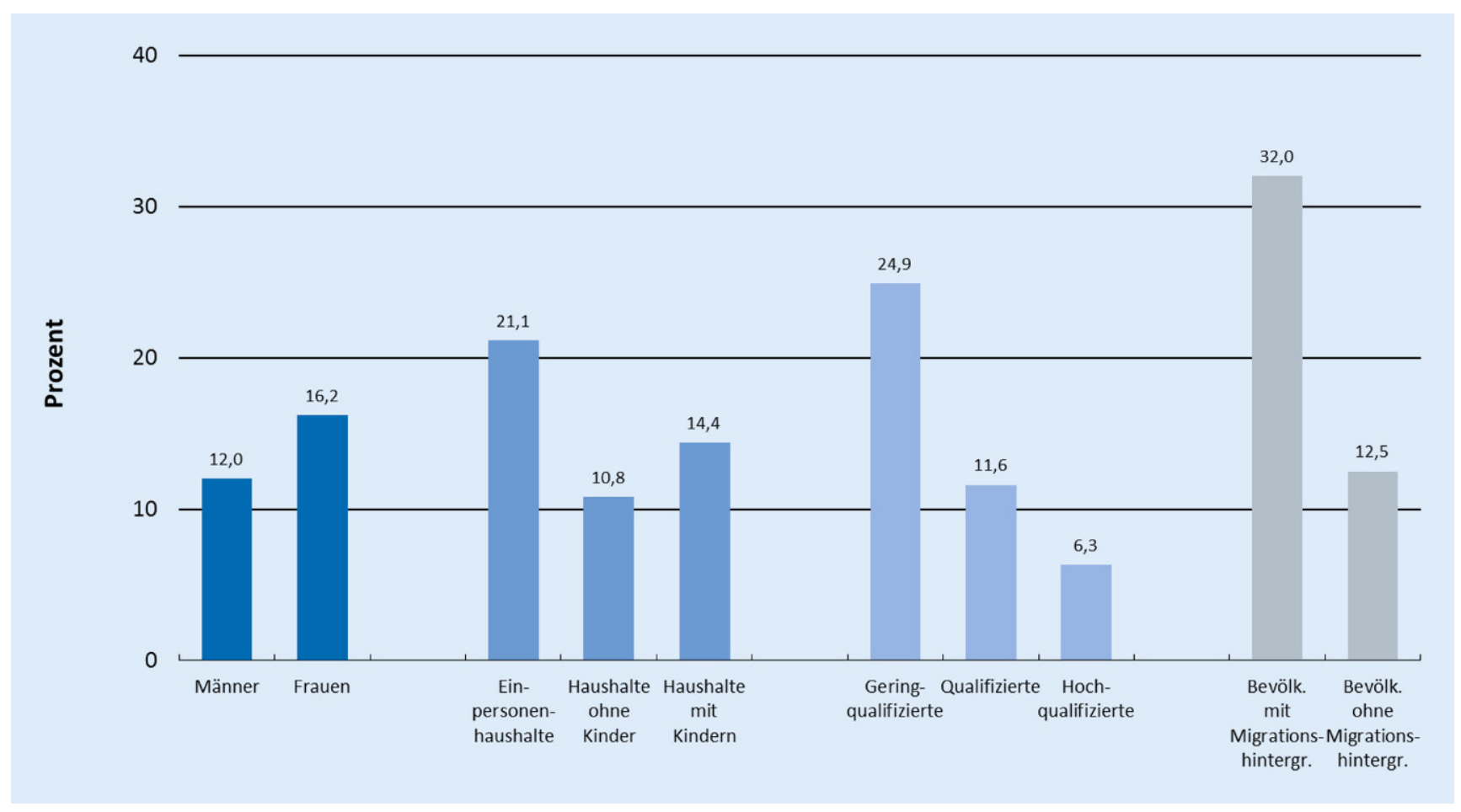

Abb. 2 ム Armutsrisiko in verschiedenen Bevölkerungsgruppen bei 65-jährigen und älteren Personen. Datenquelle: Mikrozensus 2013 [21]

stattung der Bundesregierung genutzt wird. Betrachtet wird dabei der Anteil der Personen, die ihren allgemeinen Gesundheitszustand als ,weniger gut" oder „schlecht" beurteilen und die zusätzlich in mindestens drei von fünf vorgegebenen Alltagsbereichen, z.B. beim Treppensteigen, bei der Arbeit oder bei sozialen Kontakten, „stark“ bzw. „oft“ oder ,immer" eingeschränkt sind [18]. Den Daten aus dem Jahr 2014 zufolge weisen in der Altersgruppe der 65Jährigen und Älteren 9,0\% der Männer und $10,0 \%$ der Frauen mit niedrigem Einkommen (weniger als $60 \%$ des mittleren Nettoäquivalenzeinkommens) eine weniger gute oder schlechte subjektive Gesundheit auf. Unter den Männern und Frauen mit hohem Einkommen (150\% und mehr des mittleren Nettoäquivalenzeinkommens) sind es hingegen nur $3,7 \%$ bzw. 4,2\%. Mit Blick auf die letzten 20 Jahre sprechen die SOEP-Daten dafür, dass der Anteil der Personen mit einer weniger guten oder schlechten subjektiven Gesundheit in allen Einkommensgruppen zurückgegangen ist, die einkommensbezogenen Unterschiede aber weitgehend erhalten geblieben sind (• Abb. 5).
Ergebnisse der im Ruhrgebiet durchgeführten Heinz-Nixdorf-Recall-Studie belegen, dass sozial benachteiligte Personen im Alter von 45-75 Jahren ihren allgemeinen Gesundheitszustand als schlechter einschätzen, weniger soziale Unterstützung erfahren und seltener in stabile soziale Netzwerke eingebunden sind [23, 24]. Weiterführenden Analysen zufolge kann zumindest ein Teil des Zusammenhangs zwischen sozialer Benachteiligung und subjektiver Gesundheit auf Unterschiede in den sozialen Beziehungen zurückgeführt werden [24]. Andere Studien kommen jedoch zu dem Ergebnis, dass Unterschiede in sozialen Kontakten und Beziehungen nur einen geringen Beitrag zur Erklärung der gesundheitlichen Ungleichheit im Alter leisten [25].

Soziale Unterschiede in der subjektiven Bewertung ihrer gesundheitlichen Lage lassen sich auch bei älteren Personen finden, die an mehreren Erkrankungen gleichzeitig leiden. Einer Studie zufolge, in die ausschließlich multimorbide Patienten im Alter von 65 bis 85 Jahren einbezogen wurden, weisen jene mit höherem Einkommen deutlich bessere Werte im Hinblick auf ihre subjektive Gesundheit und gesundheitsbezogene Lebensqualität sowie funktionelle Einschränkungen auf als Personen mit niedrigerem Einkommen. Diese Unterschiede bleiben auch bei statistischer Kontrolle für den ärztlich eingeschätzten Schweregrad der Erkrankungen bestehen [26]. Mit Blick auf die medizinische Versorgung zeigen bundesweite Daten zudem, dass ältere Menschen mit niedrigem sozioökonomischen Status häufiger Barrieren beim Zugang zum Versorgungssystem wahrnehmen als jene mit hohem Sozialstatus, auch wenn die Prävalenz dieser subjektiv wahrgenommenen Zugangsbarrieren insgesamt auf einem niedrigen Niveau liegt [27]. Bei Männern ab 65 Jahren lassen sich diese Unterschiede für verschiedene Leistungsbereiche beobachten, also nicht nur für ärztliche Leistungen allgemein, sondern auch für zahnärztliche Leistungen und für die Versorgung mit verordneten Medikamenten. Bei den 65jährigen und älteren Frauen waren die Unterschiede vorrangig für die Versorgung mit verordneten Medikamenten festzustellen.

In anderen europäischen Ländern sind ebenfalls soziale Unterschiede in der subjektiven Gesundheit und im 


\section{Leitthema}

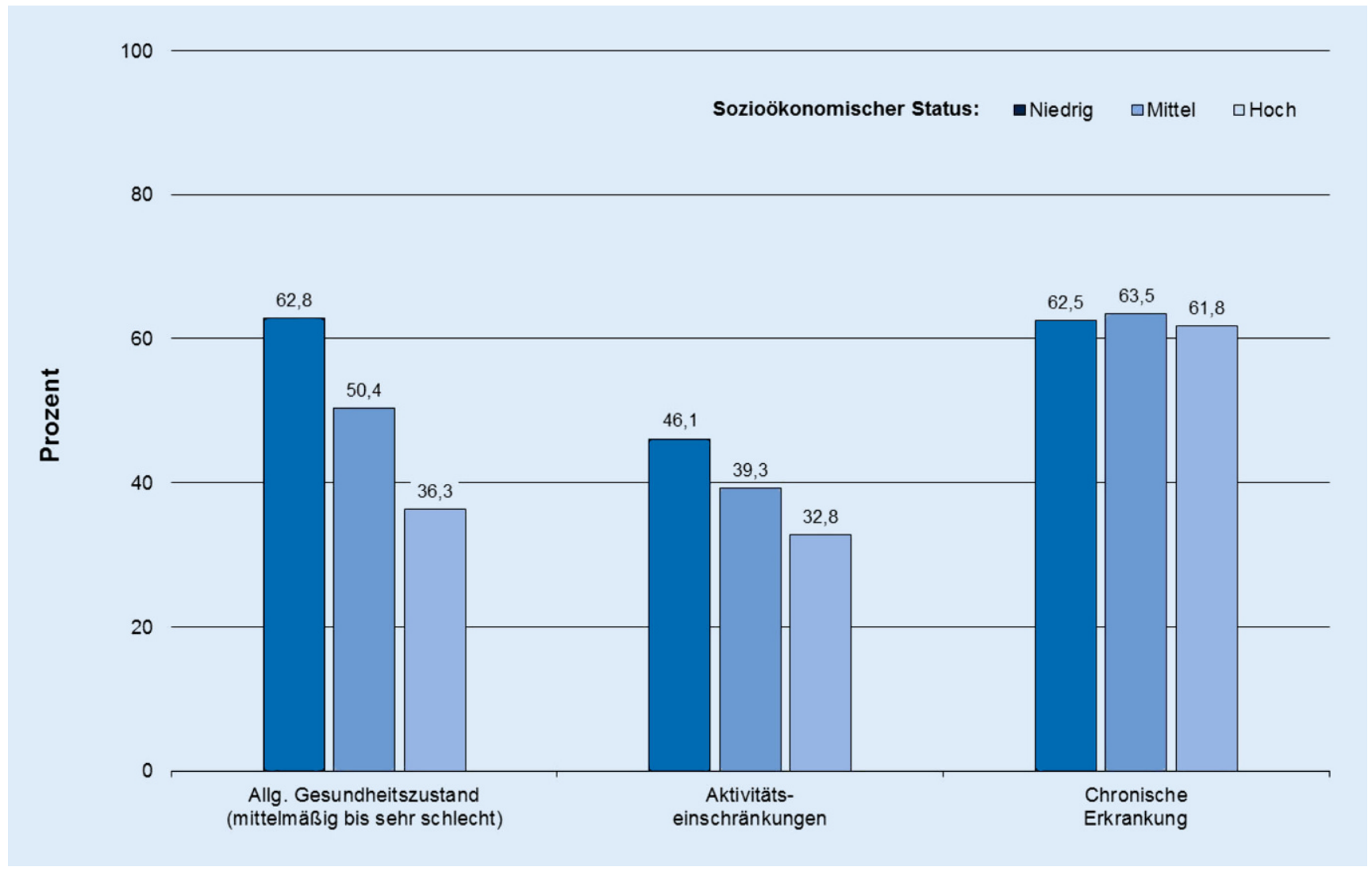

Abb. $3 \Delta$ Allgemeiner Gesundheitszustand („,mittelmäßig“, ,"schlecht" oder „,sehr schlecht"), Aktivitätseinschränkungen im Alltag (, stark" oder ",mäßig") und Vorliegen mindestens einer chronischen Erkrankung nach sozioökonomischem Status bei 65-jährigen und älteren Männern. Datenquelle: GEDA 2014/2015-EHIS

Wohlbefinden älterer Menschen zu beobachten [10]. Beispielsweise zeigen die Daten des European Community Household Panel (ECHP) und des Survey of Health, Ageing and Retirement in Europe (SHARE) übereinstimmend mit den für Deutschland vorliegenden Befunden, dass ältere Menschen aus sozial benachteiligten Bevölkerungsgruppen einen schlechteren Gesundheitszustand berichten als Personen aus vergleichsweise bessergestellten Gruppen [7, 12]. Eine aktuelle Übersichtsarbeit mit 71 Studien aus Europa bestätigt diese Befunde und ergänzt, dass sich dieses Muster nicht nur hinsichtlich der subjektiven Gesundheit, sondern in verschiedenen Bereichen von Wohlbefinden und gesundheitsbezogener Lebensqualität in älteren Bevölkerungen Europas nachweisen lässt [28].

\section{Pflegebedürftigkeit und pflegerische Versorgung}

Pflegebedürftig und leistungsberechtigt im sozialrechtlichen Sinn ist eine Person, die wegen einer körperlichen, seelischen oder geistigen Krankheit oder Behinderung für die gewöhnlichen und regelmäßig wiederkehrenden Verrichtungen des täglichen Lebens auf Dauer, voraussichtlich für mindestens sechs Monate, in erheblichem oder höherem Maße der Hilfe bedarf ( $\$ 14$ Absatz 1 SGB XI). Im Jahr 2015 waren in Deutschland etwa 2,9 Mio. Menschen als pflegebedürftig anerkannt, die Zahl der dauerhaft hilfebedürftigen Personen wird jedoch insgesamt auf vier bis fünf Millionen Personen geschätzt $[29,30]$. Mit zunehmendem Alter steigt der Anteil anerkannt Pflegebedürftiger (Pflegequote) deutlich an. Im Jahr 2015 betrug die Pflegequote in den Altersgruppen unter 75 Jahren weniger als $1,5 \%$. Unter 75 - bis 84 -Jährigen waren bereits $12,1 \%$ der Männer und 15,6\% der Frauen pflegebedürftig. Im Alter ab 90 Jahren waren etwa die Hälfte der Männer und zwei Drittel der Frauen pflegebedürftig [16].

Daten zur gesundheitlichen Lage der Pflegebedürftigen liegen aus den PflegeQualitätsberichten des Medizinischen Dienstes des Spitzenverbandes Bund der Krankenkassen (MDS) vor [31]. Diese erteilen aber keine Auskünfte zu sozioökonomischen Unterschieden in Bezug auf das Risiko der Pflegebedürftigkeit. Hierzu kann bislang nur auf vereinzelte Studien und Auswertungen von Sekundärdaten zurückgegriffen werden [32]. Beispielsweise wurden auf Basis von Daten der Gmünder Ersatzkasse biologische, soziale und krankheitsbezogene Faktoren untersucht, die Einflüsse auf das Risiko der Pflegebedürftigkeit älterer Menschen ausüben [33, 34]. Die Ergebnisse bestätigen zunächst, dass das Pflegebedürftigkeitsrisiko stark vom Lebensalter sowie vom Vorhandensein chronischer Erkrankungen und Multi- 


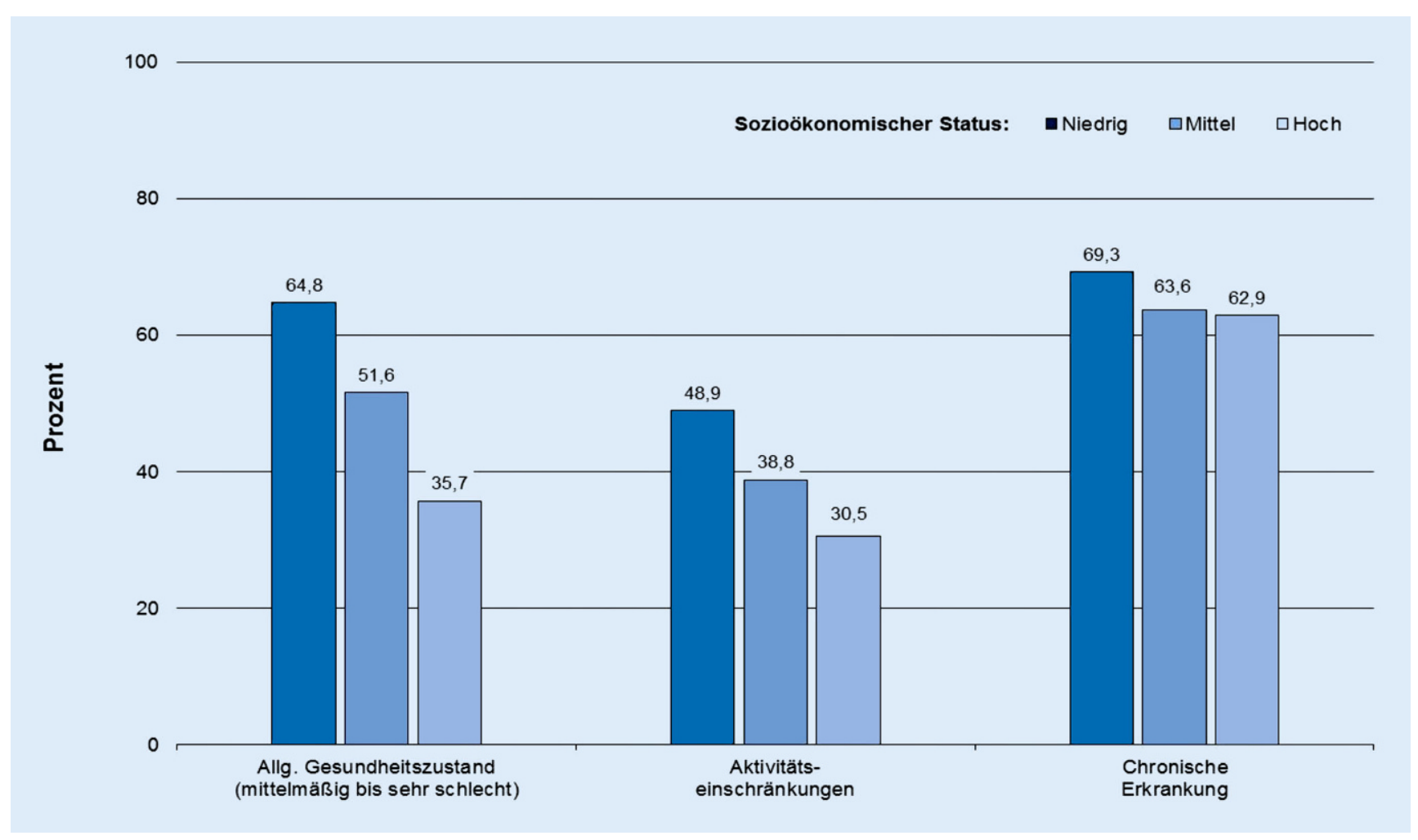

Abb. 4 ム Allgemeiner Gesundheitszustand ("mittelmäßig“, ,"schlecht" oder „sehr schlecht"), Aktivitätseinschränkungen im Alltag (",stark" oder ",mäßig") und Vorliegen mindestens einer chronischen Erkrankung nach sozioökonomischem Status bei 65-jährigen und älteren Frauen. Datenquelle: GEDA 2014/2015-EHIS

morbidität beeinflusst wird. Der Studie zufolge übt der Beruf aber auch unabhängig von diesen Faktoren einen eigenständigen Einfluss auf den Eintritt in die Pflegebedürftigkeit aus. Demnach unterliegen 45-jährige und ältere Männer, die in Arbeiterberufen tätig sind oder entsprechende Berufe während ihres aktiven Erwerbslebens ausgeübt haben, einem 22,5\% höheren Risiko für den Eintritt in die Pflegebedürftigkeit als jene aus Angestelltenberufen. Bei Frauen ist das entsprechende Pflegebedürftigkeitsrisiko für Arbeiterinnen 26,1\% höher als für Angestellte [33].

Zudem zeigt eine aktuelle Analyse mit Daten des SOEP aus den Jahren 2001 bis 2012, dass das Risiko für den Eintritt in Pflegebedürftigkeit deutlich mit der Einkommensposition zusammenhängt [35]. Den Ergebnissen zufolge ist das Pflegebedürftigkeitsrisiko von Männern und Frauen mit hohen Einkommen deutlich geringer als von jenen mit niedrigen Einkommen. Außerdem zeigte sich, dass das Vorhandensein von nahen Angehörigen die Inanspruchnahme von Pflegeleistun- gen nach SGB XI verringert. Allerdings gilt diese nur für männliche Pflegebedürftige. Die Substitution von Pflegeleistungen nach SGB XI wird also offenbar vor allem durch die Ehe- oder Lebenspartnerin geleistet. In der niedrigen Einkommensgruppe ist diese Substitution allerdings nicht zu beobachten. Hier erhöht sich sogar das Pflegerisiko, wenn eine Partnerschaft vorliegt, was die Autoren auf Kostenerwägungen und die finanzielle Anreizstruktur selber erbrachter Pflegeleistungen zurückführen [35].

Vereinzelt liegen für Deutschland auch Befunde $\mathrm{zu}$ bevorzugten Pflegearrangements in unterschiedlichen sozialen Gruppen vor. In Befragungsstudien mit 40- bis 65-Jährigen aus verschiedenen Regionen Deutschlands fand sich bei Personen mit niedrigem sozioökonomischen Status die größte Bereitschaft, die häusliche Pflege von Angehörigen auch ohne professionelle Hilfe selbst zu übernehmen [36, 37]. Die geringste Bereitschaft zur häuslichen Angehörigenpflege war bei Personen mit hohem sozioökonomischen Status festzustellen. Diese bevorzugten eher eine stationäre bzw. Heimpflege ihrer Angehörigen. Als Gründe für diese sozial unterschiedliche Verteilung von Pflegepräferenzen werden in den Studien unter anderem Kostenerwägungen angesprochen. In sozioökonomisch bessergestellten Gruppen fallen demnach Kosten, die entstehen, wenn aufgrund der Übernahme von Pflegeverpflichtungen auf attraktive berufliche und soziale Möglichkeiten verzichtet werden muss („Opportunitätskosten“), höher aus als in sozioökonomisch schlechtergestellten Gruppen. Bei Menschen mit geringen sozioökonomischen Ressourcen dürfte dagegen die relative Bedeutung der wirtschaftlichen Kosten, die mit einer Heimpflege verbunden sind, größer sein [37].

Die Daten der GEDA-Studie 2012 lassen darauf schließen, dass sich sozioökonomische Unterschiede in der Bereitschaft zur Angehörigenpflege auch in der tatsächlich geleisteten Pflege nahestehender Menschen ausdrücken. So zeigen die Ergebnisse, dass Frau- 
Tab. 1 Gesundheitsbezogene Lebensqualität („meistens" oder ",immer“ in den letzten 4 Wochen) nach sozioökonomischem Status bei 65-jährigen und älteren Männern und Frauen, in Prozent. Datenquelle: GEDA 2010 und 2012

\begin{tabular}{llll} 
& „Voller Leben“ & „Ruhig und gelassen“ & „Glücklich“ \\
\hline Männer & & & \\
Niedriger Sozialstatus & 68,4 & 67,9 & 61,1 \\
\hline Mittlerer Sozialstatus & 80,5 & 75,3 & 68,6 \\
\hline Hoher Sozialstatus & 90,3 & 87,2 & 79,4 \\
\hline Frauen & & & \\
\hline Niedriger Sozialstatus & 70,2 & 65,7 & 55,5 \\
Mittlerer Sozialstatus & 76,6 & 71,8 & 61,2 \\
Hoher Sozialstatus & 87,2 & 80,8 & 71,7
\end{tabular}

en und Männer, die mindestens zwei Stunden am Tag die Pflege nahestehender Personen übernehmen, häufiger niedrige Bildungsabschlüsse haben als jene, die keine regelmäßige Angehörigenpflege leisten [38]. Darüber hinaus belegen Daten einer Befragungsstudie aus Deutschland mit Personen, die regelmäßig in hohem Umfang Angehörige pflegen, dass der Bildungsstand von pflegenden Angehörigen bedeutsam dafür ist, ob sie Unterstützungsangebote wie professionelle Beratungsdienste und Pflegekurse oder Selbsthilfegruppen in Anspruch nehmen. Unter pflegenden Angehörigen mit hoher Bildung war die Inanspruchnahme entsprechender Angebote deutlich höher als unter Pflegenden mit niedriger Bildung [39].

Soziale Ungleichheit ist allerdings auch für professionelle Pflegekräfte von Bedeutung und nimmt Einfluss auf ihren Arbeitsalltag. Eine qualitative Untersuchung, in der Pflegekräfte ambulanter Pflegedienste interviewt wurden, veranschaulicht dies eindrücklich. Sozioökonomische Unterschiede zwischen pflegebedürftigen Personen werden durch die befragten Pflegekräfte nicht nur wahrgenommen, sie beeinflussen nach eigenen Angaben auch ihr pflegerisches Handeln und haben Auswirkungen auf den empfundenen Betreuungsumfang sowie ihre emotionale Arbeitsbelastung [40]. Die befragten Pflegekräfte beschreiben darüber hinaus auch Probleme, mit denen Pflegebedürftige mit niedriger Bildung in ihrem Alltag konfrontiert sind. Dazu gehören z. B. Schwierigkeiten beim Verstehen und Ausfüllen von Formularen zur Beantragung von Geld- und Pflegesachleistungen.

\section{Diskussion}

Die vorgestellten Ergebnisse verdeutlichen, dass die gesundheitliche Ungleichheit, die für die Bevölkerung im Erwerbsalter umfassend dokumentiert ist, auch in der älteren Bevölkerung zu beobachten ist. Sowohl in Bezug auf chronische Erkrankungen und funktionelle Einschränkungen in der Alltagsaktivität als auch Beeinträchtigungen der subjektiven Gesundheit und gesundheitsbezogenen Lebensqualität ist festzustellen, dass ältere Männer und Frauen mit niedrigem sozioökonomischen Status häufiger betroffen sind als jene mit hohem sozioökonomischen Status. Auch hinsichtlich des Risikos für Pflegebedürftigkeit und des Pflegearrangements sind Unterschiede zuungunsten der niedrigen Statusgruppe zu beobachten. Daraus ergibt sich in dieser Bevölkerungsgruppe ein besonderer Versorgungs- und Unterstützungsbedarf, der das medizinische, pflegerische und psychosoziale Versorgungssystem wie auch die sozialen Sicherungssysteme vor besondere Herausforderungen stellt.

Die für Deutschland vorliegenden Befunde entsprechen weitgehend dem internationalen Forschungsstand. Vor allem für Großbritannien, die Vereinigten Staaten und die skandinavischen Länder liegen zahlreiche Studien vor, die dafür sprechen, dass die gesundheitliche Ungleichheit auch im höheren Lebensalter deutlich ausgeprägt ist. Dies belegt z. B. ein systematisches Review $\mathrm{zu}$ sozioökonomischen Unterschieden in der Mortalität älterer Menschen, in das 44 Studien einbezogen wurden. Dass Männer und Frauen mit niedrigem so- zioökonomischen Status auch noch im fortgeschrittenen Alter ein höheres Mortalitätsrisiko haben, konnte dabei nicht nur für die allgemeine, sondern auch für die todesursachenspezifische Mortalität nachgewiesen werden [7]. Studien aus den Vereinigten Staaten, z.B. auf Basis von Daten der „Health and Retirement Study“, kommen zu ähnlichen Ergebnissen [8]. Auch in der Selbsteinschätzung des allgemeinen Gesundheitszustandes zeichnen sich in vielen Ländern bis ins höhere Lebensalter deutliche Unterschiede nach dem sozioökonomischen Status ab. In einem systematischen Review, in das 71 europäische Studien einflossen, die zwischen 1995 und 2013 publiziert wurden, konnte dargelegt werden, dass der Anteil der Älteren, die von Beeinträchtigungen des allgemeinen Gesundheitszustandes und der gesundheitsbezogenen Lebensqualität berichten, in den niedrigen Statusgruppen erhöht ist [28].

Die Befunde zur gesundheitlichen Ungleichheit im höheren Lebensalter müssen vor dem Hintergrund des demografischen Wandels gesehen und bewertet werden. Aufgrund der demografischen Entwicklung in Deutschland machen ältere Menschen einen immer größeren Anteil der Bevölkerung aus. Bisherige Untersuchungen weisen darauf hin, dass in den vergangenen Jahren alle sozioökonomischen Statusgruppen von dem Zugewinn an (gesunder) Lebenszeit profitierten, auch wenn der Zugewinn in den statusniedrigen Gruppen geringer ausfiel als in den statushöheren Gruppen [41]. Aufgrund dessen werden Personen aus sozioökonomisch benachteiligten Bevölkerungsgruppen künftig häufiger das höhere Lebensalter erreichen. Ein Teil des Rückgangs der vorzeitigen Sterblichkeit liegt darin begründet, dass immer häufiger potenziell lebensbedrohliche Krankheiten, darunter Herzinfarkt oder Diabetes mellitus, überlebt werden. Diese „Überlebenden“ leiden dann aber häufig an Folgeerkrankungen und Funktionseinschränkungen, die oftmals mit einem erheblichen Versorgungs- und Hilfebedarf einhergehen. Infolgedessen könnte die im mittleren Lebensalter $\mathrm{zu}$ beobachtende gesundheitliche Ungleichheit zunehmend bis ins höhere 

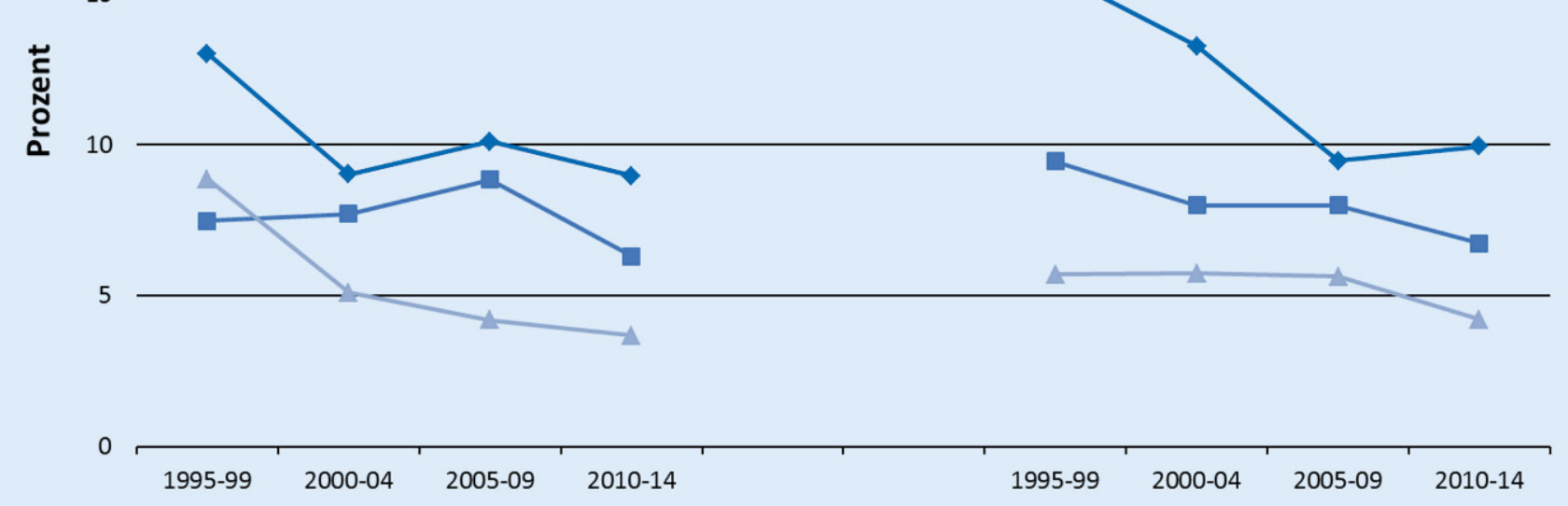

Jahr

Einkommen: $\multimap<60 \% \rightarrow 60-150 \% \multimap 150 \%$ u.m.

Abb. 5 ॥ Zeitliche Entwicklung der subjektiven Gesundheit ("weniger gut" oder „,schlecht") nach Einkommen in Prozent vom mittleren Nettoäquivalenzeinkommen in der 65-jährigen und älteren Bevölkerung. Datenquelle: SOEP 1995-2014

Lebensalter überdauern und dort in altersspezifischen Krankheiten und Behinderungen zum Ausdruck kommen. Auswirkungen des selektiven Überlebens und biologischen Alterns werden damit nicht außer Kraft gesetzt, könnten aber erst im weiter fortgeschrittenen bzw. sehr hohen Alter zu einer Verringerung der gesundheitlichen Ungleichheit führen.

Neben den Implikationen für die medizinische, pflegerische und psychosoziale Versorgung der älteren Bevölkerung ergeben sich durch die ausgeprägten sozioökonomischen Unterschiede in den Gesundheits- und Lebenschancen älterer Menschen auch Herausforderungen für die Gesundheitsförderung und Prävention. Wichtig dabei erscheint, nicht nur das Alter zu betrachten, sondern auch die vorangegangenen Lebensphasen und die Übergänge zwischen diesen, wobei mit Blick auf das Alter sicherlich dem Übergang aus dem Erwerbsleben in den Ruhestand besondere Bedeutung zukommt. Im Jahr 2012 wurde das nationale Gesundheitsziel „Gesund älter werden“ ver- öffentlicht, das sich seitdem in der Umsetzungsphase befindet [42]. Zu dessen Teilzielen zählen unter anderem, die gesellschaftliche Teilhabe älterer Menschen zu fördern, gesundheitliche Ressourcen und die Widerstandskraft älterer Menschen zu stärken und so letztlich auch die Gesundheit älterer Menschen zu verbessern, zu erhalten bzw. wiederherzustellen und Folgen von Multimorbidität zu mindern sowie Pflegebedürftigkeit vorzubeugen. Die vorgestellten Befunde zur gesundheitlichen Ungleichheit unter älteren Menschen in Deutschland bekräftigen, wie wichtig es ist, die Belange sozioökonomisch benachteiligter Gruppen dabei verstärkt in den Blick zu nehmen und diese bei der Umsetzung des Gesundheitsziels in allen Phasen und Bereichen zu berücksichtigen.

Wie auch in früheren Lebensphasen eignen sich verhältnisbezogene Ansätze auch bei Älteren besonders für Gesundheitsförderung und Prävention in benachteiligten Gruppen, weil die Lebensverhältnisse einerseits eigenstän- dige Gesundheitsrelevanz haben, aber andererseits auch gesundheitsrelevante Verhaltensweisen beeinflussen können. Ein exemplarisches Setting für verhältnisbezogene Maßnahmen bei Älteren ist die unmittelbare Wohnumgebung, da besonders sozial schlechtergestellte Menschen im höheren Alter in ihrer Mobilität, z. B. hinsichtlich Reisen und überregionaler Kontakte, auch aus finanziellen Gründen eingeschränkt sind [42]. Zudem wird der räumliche Aktionsradius älterer Menschen durch altersbedingte Funktionseinschränkungen zunehmend kleiner. Wie die dargestellten Forschungsergebnisse zeigen, sind Funktionseinschränkungen unter älteren Menschen aus sozial benachteiligten Gruppen besonders verbreitet. Die Gestaltung der unmittelbaren Wohnumgebung älterer Menschen unter Berücksichtigung altersgerechter Bedarfe und Bedürfnisse stellt daher einen zentralen lebensweltbezogenen Ansatz dar, um die Teilhabechancen sozial schlechtergestellter Menschen im höheren Alter zu verbessern. 


\section{Korrespondenzadresse}

\section{PD Dr. Thomas Lampert}

Fachgebiet Soziale Determinanten der Gesundheit, Robert Koch-Institut General-Pape-Str. 62-66, 12101 Berlin, Deutschland

lampertt@rki.de

\section{Einhaltung ethischer Richtlinien}

Interessenkonflikt. T. Lampert und J. Hoebel geben an, dass kein Interessenkonflikt besteht.

Dieser Beitrag beinhaltet keine von den Autoren durchgeführten Studien an Menschen oder Tieren.

Open Access. Dieser Artikel wird unter der Creative Commons Namensnennung 4.0 International Lizenz (http://creativecommons.org/licenses/by/4.0/deed. de) veröffentlicht, welche die Nutzung, Vervielfältigung, Bearbeitung, Verbreitung und Wiedergabe in jeglichem Medium und Format erlaubt, sofern Sie den/die ursprünglichen Autor(en) und die Quelle ordnungsgemäß nennen, einen Linkzur Creative Commons Lizenz beifügen und angeben, ob Änderungen vorgenommen wurden.

\section{Literatur}

1. Gruenberg EM (1977) The failures of success. Milbank Mem Fund Q Health Soc 55:3-24

2. Olshansky SJ, Ault AB (1986) The fourth stage of the epidemiologic transition: the age of delayed degenerative diseases. Milbank Q 64(3):355-391

3. Fries JF (1980) Aging, natural death, and the compression of morbidity. NEnglJMed 303(3):130-135

4. Fries JF (2003) Measuring and monitoring success in compressing morbidity. Ann Intern Med 139(5Part_2):455

5. Lampert T, Kroll LE, Müters S, Stolzenberg H (2013) Messung des sozioökonomischen Status in der Studie "Gesundheit in Deutschland aktuell" (GEDA). Bundesgesundheitsbl Gesundheitsforsch Gesundheitsschutz 56(1):131-143

6. von dem Knesebeck O, Vonneilich N (2009) Gesundheitliche Ungleichheit im Alter. Z Gerontol Geriatr 42(6):459-464

7. Huisman M, Read S, Towriss CA et al (2013) Socioeconomic inequalities in mortality rates in old Age in the world health organization europe region. Epidemiol Rev 35(1):84-97

8. Shaw BA, McGeever K, Vasquez E et al (2014) Socioeconomic inequalities in health after age 50 : are health risk behaviors to blame? Soc Sci Med 101:52-60

9. Fors S, Thorslund M (2015) Enduring inequality: educational disparities in health among the oldest old in Sweden 1992-2011. Int J Public Health 60(1):91-98

10. von dem Knesebeck O, Schäfer I (2009) Gesundheitliche Ungleichheit im höheren Lebensalter. In: Richter M, Hurrelmann K (Hrsg) Gesundheitliche Ungleichheit. VS, Wiesbaden, S253-265

11. Schöllgen I, Huxhold O, Tesch-Römer C (2010) Socioeconomic status and health in the second half of life: findings from the German Ageing Survey. Eur J Ageing 7(1):17-28
12. Leopold L, Engelhardt H (2013) Education and physical health trajectories in old age. Evidence from the Survey of Health, Ageing and Retirement in Europe (SHARE). Int JPublic Health 58(1):23-31

13. Markides KS, Machalek R (1984) Selective survival, aging and society. Arch Gerontol Geriatr 3(3):207-222

14. Dowd JJ, Bengtson VL (1978) Aging in minority populations. An examination of the double jeopardy hypothesis. J Gerontol 33:427-436

15. Lampert T (2009) Soziale Ungleichheit und Gesundheit im höheren Lebensalter. In: Robert Koch-Institut (Hrsg) Gesundheit und Krankheit im Alter. Robert Koch-Institut, Berlin

16. Statistisches Bundesamt (2018) Pflegestatistik Ambulante und stationäre Pflegeeinrichtungen: Grunddaten, Personalbestand, Pflegebedürftige, Empfänger und Empfängerinnen von Pflegegeld leistungen

17. Statistisches Bundesamt (2015) Bevölkerung Deutschlands bis 2060. Ergebnisse der 13. koordinierten Bevölkerungsvorausberechnung. DeStatis, Wiesbaden

18. BMAS - Bundesministerium für Arbeit und Soziales (2018) Armuts- und Reichtumsbericht. https://www.armuts-und-reichtumsbericht.de/ DE/Startseite/start.html.Zugegriffen: 8. Okt. 2018

19. Goebel J, Grabka MM (2011) Zur Entwicklung der Altersarmut in Deutschland. Diw Wochenbericht 25:1-16

20. Bertelsmannstiftung (2017) Entwicklung der Altersarmut bis 2036 Trends, Risikogruppen und Politikszenarien. https:// www.bertelsmann-stiftung.de/fileadmin/ files/BSt/Publikationen/GrauePublikationen/ Entwicklung_der_Altersarmut_bis_2036.pdf. Zugegriffen: 8. Okt. 2018

21. Bertelsmannstiftung (2015) Altersarmut in Deutschland - regionale Verteilung und Erklärungsansätze. Analysen und Konzepte aus dem Programm "LebensWerte Kommune", Ausgabe 4/2015. https://www.bertelsmannstiftung.de/fileadmin/files/BSt/Publikationen/ GrauePublikationen/Policy_LebensWK_Okt_ 2015_final.pdf.Zugegriffen: 8. Okt. 2018

22. Lampert T, Hoebel J, Kuntz B, Müters $S$, Kroll LE (2017) Gesundheitliche Ungleichheit in verschiedenen Lebensphasen. Beiträge zur Gesundheitsberichterstattung des Bundes. Gemeinsam getragen von RKI und Destatis. Robert KochInstitut, Berlin

23. Weyers S, Dragano N, Mobus S et al (2008) Low socio-economic position is associated with poor social networks and social support: results from the Heinz Nixdorf Recall Study. Int J Equity Health 7:13

24. Vonneilich N, Jöckel KH, Erbel R et al (2012) The mediating effect of social relationships on the association between socioeconomic status and subjective health-results from the Heinz Nixdorf Recall cohort study. BMC Public Health 12:285

25. von dem Knesebeck O (2005) Die Bedeutung sozialer Beziehungen für den Zusammenhang zwischen sozialer Ungleichheit und Gesundheit im Alter. Soz Praventivmed 50(5):311-318

26. von dem Knesebeck O, Bickel H, Fuchs A et al (2015) Social inequalities in patient-reported outcomes among older multimorbid patients-results of the MultiCare cohort study. Int JEquity Health 14(1):17

27. Hoebel J, Rommel A, Schröder SL, Fuchs J, Nowossadeck E, Lampert T (2017) Socioeconomic inequalities in health and perceived unmet needs for healthcare among the elderly in Germany. Int J Environ Res Public Healt 14:10
28. Read S, Grundy E, Foverskov E (2016) Socioeconomic position and subjective health and well-being among older people in Europe: a systematic narrative review. Aging Ment Health 20(5):529-242

29. BMFSFJ (2010) Sechster BerichtzurLage derälteren Generation in der Bundesrepublik Deutschland - Altersbilder in der Gesellschaft. Bundesministerium für Familie, Senioren, Frauen und Jugend (BMFSFJ), Berlin

30. Robert Koch-Institut (Hrsg) (2015) Gesundheit in Deutschland. Gesundheitsberichterstattung des Bundes. Gemeinsam getragen von RKI und Destatis. RKI, Berlin

31. MDS (2014) Vierter Pflege-Qualitätsbericht des MDS. Qualität in der ambulanten und stationären Pflege.MedizinischerDienst des Spitzenverbandes Bund der Krankenkassen e. V. (MDS), Essen

32. Kruse A, Schmitt E (2016) Soziale Ungleichheit, Gesundheit und Pflege im höheren Lebensalter. Bundesgesundheitsbl Gesundheitsforsch Gesundheitsschutz 59(2):252-258

33. Borchert L (2008) Soziale Ungleichheit und Gesundheitsrisiken älterer Menschen: Eine empirische Längsschnittanalyse unter Berücksichtigung von Morbidität, Pflegebedürftigkeit und Mortalität. MaroVerlag, Augsburg

34. Borchert L, Rothgang H (2008) Soziale Einflüsse auf das Risiko der Pflegebedürftigkeit älterer Männer. In: Bauer U, Büscher A (Hrsg) Soziale Ungleichheit und Pflege: Beiträge sozialwissenschaftlich orientierter Pflegeforschung. VS, Wiesbaden, S215-237

35. Unger R, Giersiepen K, Windzio M (2015) Pflegebedürftigkeit im Lebensverlauf: Der Einfluss von Familienmitgliedern und Freunden als Versorgungsstrukturen auf die funktionale Gesundheit und Pflegebedürftigkeit im häuslichen Umfeld. KzfSS67(1):193-215

36. Blinkert B (2005) Pflege und soziale Ungleichheit - Pflege und "soziale Milieus". In: Schroeter KR, Rosenthal T (Hrsg) Soziologie der Pflege: Grundlagen, Wissensbestände und Perspektiven. Juventa, Weinheim, S141-156

37. Blinkert B, Klie T (2008) Soziale Ungleichheit und Pflege. Polit Zeitgesch 12-13:25-33

38. Wetzstein M, Rommel A, Lange C (2015) Pflegende Angehörige - Deutschlands größter Pflegedienst. GBE kompakt 6(3):1-11

39. Lüdecke D, Mnich E, Kofahl C (2012) The impact of sociodemographic factors on the utilisation of support services for family caregivers of elderly dependents: results from the German sample of the EUROFAMCARE study. Psychosoc Med 9:Doc6

40. Möller A, Osterfeld A, Büscher A (2013) Soziale Ungleichheit in der ambulanten Pflege. Z Geronto Geriatr 46(4):312-316

41. Unger R, Schulze A (2013) Können wir (alle) überhaupt länger arbeiten? Trends in der gesunden Lebenserwartung nach Sozialschicht in Deutschland. Comparative Population Studies. ZBevolkerungswiss 38(3):545-564

42. Kooperationsverbund gesundheitsziele.de (2012) Nationales Gesundheitsziel: Gesund älter werden. Bundesministerium für Gesundheit, Berlin 